\title{
Una aplicación de la medición de la productividad científica de los investigadores en organizaciones universitarias cubanas
}

\author{
Walfredo González Hernández \\ Universidad de Matanzas, Cuba \\ Maritza Petersson Roldán \\ Universidad de Matanzas, Cuba \\ Yonelkys Ramos Mena \\ Universidad de Matanzas, Cuba
}

\section{ORIGINAL}

\begin{abstract}
Resumen
Objetivo. El artículo presenta un análisis de la evaluación de la producción científica y los indicadores que la miden. Posteriormente se presenta una métrica que modela de forma cuantitativa los indicadores de producción científica que se utilizan en las universidades cubanas en forma de función aditiva en función del tiempo que dedican los investigadores.

Método. Por último, se aplica parcialmente la métrica en la Universidad de Matanzas y se analizan los datos obtenidos.

Resultados. Los resultados permiten diferenciar de manera cualitativa y cuantitativa a los investigadores de esta universidad atendiendo a sus resultados de investigación en el documento oficial de la universidad.

Conclusiones. La métrica constituye una herramienta para la toma de decisiones en cuanto a la organización de los recursos humanos que posee la universidad en función de la investigación.
\end{abstract}

Palabras clave:

Evaluación de la ciencia; Métrica; Productividad científica.

\section{The measurement of scientific productivity of investigators in Cuban university}

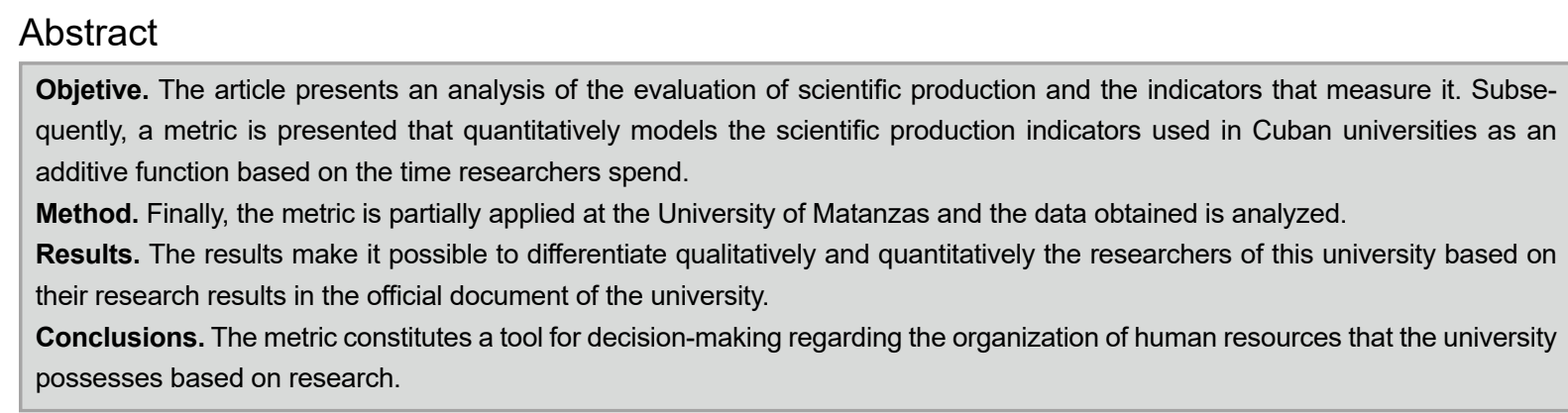

Keywords:

Metrics; Science evaluation; Scientific productivity.

\section{Introducción}

La investigación científica es uno de los procesos más importante de las universidades pues les corresponde a estas la producción de conocimiento como parte de su encargo social. De ahí que las universidades dediquen esfuerzos y recursos humanos a la producción de conocimientos. Sin embargo, la gestión de estos recursos humanos a partir de los procesos sustantivos puede tener sesgos subjetivos a partir de las creencias sobre la producción científica que estas poseen. De la misma manera, es imprescindible que las personas sean valoradas por sus resultados en función del tiempo que la organización le asigna a la producción científica. De los sistemas de evaluación de la ciencia, el sexenio español (Deusto, 2018) considera como importante las publicaciones en los dos primeros cuartiles o en la base de datos de reconocido prestigio aunque la cantidad de publicaciones por investigador varía en dependencia del área del conocimiento. Se reconoce que existen elementos que penalizan la producción científica entre los cuales se destacan reiteración de publicaciones en revistas de la organización a la cual pertenece el investigador y cuando no se justifique el número de investigadores como autores del artículo. 
Las universidades cubanas mantienen otros indicadores de medición de la ciencia pues las publicaciones se han dividido cuatro grupos y cada uno de ellos tienen diferente nivel de importancia. Otros indicadores que se tienen en cuenta son las tutorías de tesis, los premios obtenidos y otras, pero con niveles diferentes de atención respecto a las publicaciones. Sin embargo, en las universidades se pueden instrumentar otros indicadores para medir el impacto de los investigadores como el índice h, h10 y RG. Al igual que el resto de los países, las universidades cubanas necesitan despojándose, en la medida de lo posible, de la subjetividad inherente a estos procesos, lo que hace necesario disponer de una métrica que permita cuantificar esta producción. Precisamente el objetivo de este trabajo es diseñar una métrica que permita una evaluación objetiva de la productividad de los investigadores utilizando como información de base los resultados de los profesores-investigadores en los indicadores relativos a la investigación científica.

\section{Revisión de la Literatura}

La medición es un proceso inherente a la evaluación de los procesos que pueden resultar estratégicos en una organización. Las universidades como organizaciones educativas poseen tres procesos considerados sustantivos: académico, laboral e investigativo. Dentro de los procesos investigativos existen varios indicadores que para algunos autores (Aguillo Caño, Uribe Tirado, \& López López, 2017) son importantes utilizar como los indicadores de GoogleScholar (total de citas, h, i10) y el de ResearchGate (RG) y recomiendan utilizar scimago aunque se reconoce el potencial de RG como indicador del impacto de las producciones científicas. Estos indicadores son importantes, aunque en el caso de RG puede ser falseado a partir de las autocitas y documentos que no revelan su origen. Además, el autor puede no revelar la fuente de su estudio o remitir a las revistas pago, lo cual no habla de un acceso abierto a estos documentos científicos. Otro estudio de la excelencia en las investigaciones define qué se entiende por este concepto (Ferretti, Pereira, Vértesy, \& Hardeman, 2018). Para ello se basa en los siguientes indicadores:

- Debe evocar un sentido general de valía y, con ello, compartibilidad.

- Muchos de sus elementos no son susceptibles de ser medidos de manera directa.

- Diferentes puntos de vista sobre la ciencia y su contenido.

A continuación estos autores (Ferretti et al., 2018)se realizan tres preguntas interesantes alrededor de cómo medir la productividad científica y asumen los indicadores de investigación de excelencia (RES\&T), pero en su gran mayoría se asumen las publicaciones indexadas en WOS o Scopus por lo que son indicadores restrictivos. Para otro autor (Handley Jones, 2017) es importante incluir en estas mediciones los arbitrajes de artículos así como los libros publicados pues son forma de producción de la ciencia aunque en los indicadores asumidos por otros autores no sean mencionados.

Una crítica a los indicadores dominantes como factor de impacto y otros se asumen en otras investigaciones como (Manzano-Arrondo, 2017). Este autor analiza las problemáticas que generan el uso de estos indicadores para la evaluación de la ciencia al mismo tiempo que generan ciclos de exclusión. Para ello propone varias propuestas. Una primera al plantear que se realicen matizaciones al modelo:

\footnotetext{
1) corregir sesgos de cobertura regional, de idioma y de disciplina; 2) fomentar el acceso abierto al contenido íntegro de las revistas consideradas, y 3) manejar un conjunto extenso y transparente de criterios científicos de inclusión de revistas en la base de datos" (Manzano-Arrondo, 2017, p. 14),
}

Aunque se asume que las tutorías pudieran ser también de trabajos de diploma y maestría que, aunque de menor rigor que el doctorado, pero son resultados científicos válidos. Una segunda relacionada con el cambio de perspectiva: declaraciones de principios dora e iniciativas previas donde:

\footnotetext{
la $3 a$ es considerada ya en algunas pautas de evaluación, donde se han incluido la dirección de tesis doctorales, la generación de patentes, etc., a lo que puede añadirse la consideración de formatos abiertos como documentos pdf, páginas web, blogs o vídeos (Manzano-Arrondo, 2017, p. 15)
}

Utilizar métodos cualitativos que incluyan elementos como el impacto social y la mayor variedad posible de resultados de la ciencia. En esta última propuesta es interesante para este análisis, desde el artículo, considerar que el impacto social es importante pues no se produce la ciencia sólo para recrear la realidad y extraer de ella conocimiento sino además para aplicarla a la solución de las problemáticas que les dio origen, así como generalizarlas de ser posible. En estas expresiones no se asumen posiciones excluyentes con las investigaciones que permiten explorar lo singular como fuente generadora de conocimiento y se reconoce el carácter complejo de la investigación científica. Para este propósito el autor Manzano-Arrondo (2017) asume ciertos principios rectores entre los cuales se encuentra ampliar el concepto de productividad científica y se asume cuando

. la medida de los impactos políticos y sociales. Esta dificultad no merma su pertinencia. Muy al contrario, constituye la preocupación más creciente y relevante" (Manzano-Arrondo, 2017, p. 21). 
Otro acercamiento a los indicadores de medición de la ciencia en la universidad propuesto por los autores Biava, Pagani, and de Oliveira (2019) y propone como indicadores para medir la producción científica los siguientes: Artículo publicado en eventos, trabajo publicado en memorias de eventos, capítulo de libros, artículo aceptado para publicar, otra producción bibliográfica, libros publicados, entre otros. Sin embargo, de este autor no se tienen en cuenta otros aspectos ya señalados como es el impacto que tienen las investigaciones en la sociedad, así como una relación entre estos indicadores que permita cualificar la productividad de los investigadores. Un acercamiento interesante a esta temática lo propone Kosten (2016) al proponer un conjunto de indicadores para la organización clasificados en cuatro grupos principales: políticas generales de la ciencia, asignación de fondos, organización y dirección, gestión de contenidos y decisiones y consumo de información. Estos grupos son necesarios e importantes para la producción de una organización, sin embargo, no permiten cua cada investigador. Por su lado, Aguillo (2015) propone métricas de la web 2.0 que son importantes pues miden el posicionamiento en redes sociales para científicos. En esta conferencia el autor aborda varios de los perfiles de usuarios como ResearcherID, ResearchGate, perfil de scholar que aportan índices del impacto que tienen los artículos para una comunidad científica además que propone la combinación de varias métricas para evaluar la ciencia en la web. De análisis de estos investigadores se desprende que toda producción científica debe ser tenida en cuenta, aunque de cada una de ellas hay sus clasificaciones y formas de medición.

Generalmente los países poseen un sistema de clasificación por grupos de las revistas como indicador de la valoración de la importancia que tienen cada una de ellas, entre otras cuestiones. Cuba clasifica los grupos en 4 donde ordena por el nivel de importancia de las revistas priorizando las revistas de mayor visibilidad mientras que México no distingue grupos y se publican aquellas revistas en el Índice de Revistas Mexicanas de Divulgación Científica y Tecnológica (Conacyt, s/f). Por su parte, Colombia elaboró un sistema de principios (Caballero-Uribe \& Viloria-Doria, 2018): transición de la gestión editorial a impacto científico; valoración del impacto por áreas del conocimiento; acceso abierto; métricas-cálculo para medir el impacto y la política editorial institucional que les permite obener una clasificación dividida en 4 grupos de mayor a menor importancia. De estas clasificaciones se infiere que los países otorgan mayor importancia a unas revistas que otras, por lo que debe tenerse en cuenta como factor de ponderación de la importacia que tiene para los países, y consecuentemente en las universidades, de cada artículo publicado. De la misma manera con los proyectos y las tutorías de tesis. Sin embargo, las instituciones no siempre tienen en cuenta y valoran de la misma manera la producción de artículos, las tutorías de tesis y la participación en proyectos y los arbitrajes que se realizan a los artículos de las revistas. Así mismo, un factor que debe ser tenido en cuenta es la ocupación de la persona, pues aquel que su trabajo es publicar pues no realiza el mismo esfuerzo que aquel que ocupa otras funciones dentro de la organización por lo que no puede dedicarle tiempo completo a ello. Por ello cada ítem debe llevar un factor de peso por cada categoría de la producción científica, así como otro factor de ponderación que valora cuán importante es el ítem para la organización. Es posible que las universidades no valoren la participación en eventos o el arbitraje de revistas como la publicación de artículo. Sin embargo, estas consideraciones quedarían en análisis cualitativos interesantes y necesarios pero que pudieran perderse en las consideraciones subjetivas de quienes lo realizan. Las métricas como relaciones cuantitativas de medidas establecen valores numéricos que permiten asignar un valor cuantitativo a relaciones que pudieran ser cualitativas. Para Gorraiz (2018)

$$
\begin{aligned}
& \text { "Existen dos tendencias divergentes en cuanto al uso práctico de las nuevas métricas, como muy bien } \\
& \text { muestran dos de los productos más usados a este respecto: Altmetric.com y PlumX" (p. 233). }
\end{aligned}
$$

En la investigación propuesta por (Gumpenberger, Glänzel, \& Gorraiz, 2016) se plantea que siguiendo al sitio web Altmetric.com, ellos proveen una puntuación como medida cuantitativa que se compone de la atención que ha recibido un artículo académico y se basa en tres factores principales:

1. Volumen: el puntaje de un artículo aumenta a medida que más personas lo mencionan (restricción: solo se cuenta una mención de cada persona por fuente).

2. Fuentes: están categorizadas y cada categoría de mención contribuye de manera diferente a la puntuación final (hay una tabla con los diferentes pesos disponibles).

3. Autores: aquí cuenta quién y con qué frecuencia y a quién alguien menciona algo.

Por su lado, al decir de Ortega (2018), PlumX incluye varias métricas de citas y de uso de la información, aunque solamente trabaja redes sociales y según este autor abarca tres grandes bases de datos: EBSCO, Elsevier y Scopus. Sin embargo, en estas métricas propuestas aún no se evidencia una relación cuantitativa de la cual emerja todas las posibles expresiones de la producción científica que está recogida en varias web para la conformación de currículos, una de ellas es el Currículo Lattes desarrollado por la CNPq de Brasil (http://www.cnpq.br/). En las métricas analizadas propuestas por varias investigaciones (Aguillo, 2015; Biava et al., 2019; Dorta-Contreras, 2018; Ferretti et al., 2018; Gumpenberger et al., 2016) las nuevas métricas adolecen de varias insuficiencias:

1. No abarcan una amplia variedad de formas de producción científica que permitan establecer relaciones cuantitativas que caractericen mejor al investigador.

2. Se basan en bases de datos criticadas (Handley Jones, 2017) por su carácter cíclico y elitista. 
3. No se realizan ponderaciones que permita a las organizaciones tener flexibilidad en la evaluación de aquellos aspectos que les resulte más interesante a ellas ponderar en un momento determinado con mayor importancia que otros.

4. Generalmente se mide el impacto en la comunidad investigadora pero no el impacto que causa en la sociedad.

Tomando en cuenta los elementos analizados hasta el momento se plantea como métrica PT: productividad científica del trabajador a la siguiente ecuación:

$$
\begin{gathered}
P T=\frac{1}{\text { Tiemp }} \times\left[p_{\text {puótic }} \times \operatorname{PrA}+p_{\text {proyec }} \times \operatorname{Pr} P+p_{\text {resis }} \times \operatorname{Pr} T+p_{\text {premio }} \times \operatorname{Pr} R+p_{\text {event }} \times \operatorname{PrE}+p_{\text {aró }} \times \operatorname{PrArb}+p_{\text {lio }}\right. \\
\left.\times \text { PrLib }+0_{\text {ind }} \times \text { Otros }\right] \quad \text { (ecuación 1) }
\end{gathered}
$$

Donde:

Tiemp: Tiempo dedicado a la investigación en el período evaluado (mayor que cero), $P_{\text {public }}, P_{\text {proyec }}, P_{\text {tesis, }}, P_{\text {premio }}, P_{\text {event }}$, $P_{a r b}, P_{l i b}, O_{i n d}$ : pesos concedidos a las actividades de la investigación: publicación, proyecto, tesis, premio, evento, arbitrajes y libros y otros respectivamente mientras que $\operatorname{Pr} A$ : Producción de artículos en el período evaluado, $\operatorname{Pr} P$ : Producción en proyecto, PrT: Producción en tesis, PrR: Producción en premios, PrE: Producción en evento, PrArb: Número de arbitrajes que produce para congresos o revistas, PrLib: Producción de Libros o Capítulos de Libros y $O_{\text {ind }}$ : Producción en otros indicadores asociados a la actividad científica que la institución considere.

A continuación, se exponen las expresiones de cálculo de cada una de estas componentes:

- PrA: Producción de artículos en el período evaluado

$$
\operatorname{PrA}=\sum_{i=1}^{n}\left(\sum_{j=1}^{m_{i}} A r t_{i j}\right) a_{i}
$$

Donde $a_{i}$ : peso otorgado por la institución a las publicaciones del grupo $i$. En el caso cubano se reconocen cuatro grupos, por tanto, $n=4$, donde la importancia aumenta en el decrecimiento de la escala, $m_{i}$ : Total de publicaciones del grupo $i$ en el período evaluado y $A_{r i j}$ : Participación del trabajador (profesor o investigador) en la publicación $j$ del grupo $i$ y se determina con la expresión siguiente:

$$
\operatorname{Art}_{i j}=\frac{1}{\text { cantidad de autores de la publicación } j \text { del grupo } i}\left(f_{\text {complejidad externa }} / f_{\text {ayuda insemal }}\right)
$$

- PrP: Producción en proyecto en el período evaluado

$$
\operatorname{Pr} P=\sum_{i=1}^{k}\left(\sum_{j=1}^{\hat{h}_{j}} \text { proyectos }_{i j}\right) \text { p_proyecto }
$$

Donde $p$ proyecto $_{i}$ : Peso otorgado por la institución a los proyectos del tipo $i$. El tipo está dado según la categoría de los proyectos en internacionales, nacionales, empresariales, institucionales u otros que surjan, por lo que $k$ es el total de categorías, $h_{i}$ : Total de proyectos de tipo $i$ en que participa el trabajador y proyectos p $_{i j}$ Participación del trabajador en el proyecto $j$ del tipo $i$. A continuación, se expone la expresión de cálculo de dicho indicador:

$$
\text { proyectos }_{i j}=\frac{\text { número de tareas en el proyecto } j \text { del tipo } i-\text { número de tareas evaluadas }}{\text { total de tareas del proyecto } j \text { del tipo } i}
$$

número de tareas evaluadas: indica la cantidad de tareas que generaron alguno de los indicadores que son tomados en consideración por otros como artículos publicados, presentados a eventos, entre otras.

- PrT: Producción en tesis

$$
\operatorname{Pr} T=\sum_{i=1}^{a}\left(\sum_{j=1}^{b_{1}} \text { NTesis }_{i j}+N \text { Trib }_{i}+N O p O n_{i}\right) \times p_{-} \text {tesis }_{i}
$$

Donde $p_{\text {_tesis }}$ : peso otorgado por la institución al tipo de tesis $i$, considerando si la tesis es doctorado, maestría, especialidad, diploma u otras que surjan, por lo que a es el total de tipo de tesis, $b_{t}, c_{t}, d_{t}$. Total de Tesis de tipo $i$ en que ha intervenido el trabajador en calidad de tutor, tribunal u oponente respectivamente, $N T e s i s_{i j}:=\%$ de responsabilidad con la tesis $j$ del tipo $i, N$ Trib $_{i}$ : Total de participaciones en tribunales de tesis del tipo $i, N$ ppon $_{i j}$ : Total de oponencias realizadas de tesis de tipo i, $\operatorname{PrR}$ : Producción en premios.

$$
\operatorname{Pr} R=\sum_{\mathrm{i}=1}^{\mathrm{r}}\left(\sum_{\mathrm{j}=1}^{p_{1}} \operatorname{Npremios}_{\mathrm{i} j}\right) p r_{\mathrm{i}}
$$


Donde $p r_{i}$ : Peso concedido por la institución a cada grupo de clasificación de los premios que pueden ser de internacionales, academia, CITMA u otras que surjan, por tanto, $t$ es el total de tipos de premios, $p i$ : Total de premios de tipo $i$ en que ha participado el trabajador, $\operatorname{Npremios}_{i j}$ : \% de participación en el premio $j$ del tipo $i, \operatorname{Pr} E$ : Producción en evento.

$$
\operatorname{PrE}=\sum_{i=1}^{\ell}\left(\sum_{j=1}^{q_{1}} \text { Ponencias }_{i j}-A r \tau_{i j}+\% \text { reutilización }\right) p_{-} \text {even }
$$

Donde $p$ event $:$ : Peso otorgado al evento $i$ por la institución atendiendo la categoría del evento: internacionales, nacionales, provinciales, municipales, universitarios u otros que considere la organización, qi: Total de eventos en que ha participado el trabajador en eventos de tipo $i$, Ponencias $s_{i j}$ : \% en la autoría de la ponencia $j$ en evento de tipo $i$, y se determina con la siguiente expresión:

Ponencias $i j=\frac{1}{\text { total de autores de la potencia } j \text { en el evento de categoría } i}$

- PrArb : Arbitraje de artículos en el período evaluado

$$
\text { PrArb }=\sum_{i=1}^{n}\left(\sum_{j=1}^{m_{i}} A r t A r b_{i j}\right) a r_{i} \times p_{a c i e r t c}
$$

Donde $a r_{i}$ : peso otorgado por la institución a las publicaciones del grupo $i$. En el caso cubano se reconocen cuatro grupos, por tanto $n=4$, donde la importancia aumenta en el decrecimiento de la escala. Para ellos se pueden asumir la misma escala que en el caso de los artículos, $m_{i}$ : Total de publicaciones del grupo $i$ en el período evaluado, $A_{r t A r b}$ : Participación del trabajador (profesor o investigador) en el arbitraje de la publicación $j$ del grupo $i$, Pacierto $_{i}$ : porcentaje de evaluaciones positivas o negativas respecto a la decisión final y PrLib : Producción de artículos en el período evaluado.

$$
\operatorname{PrLib}=\sum_{i=1}^{n}\left(\sum_{j=1}^{n_{i}} L_{i b r o s}\right)
$$

Donde $a_{i}$ : peso otorgado por la institución a los libros publicados del grupo $i$. En el caso cubano se reconocen cuatro grupos, por tanto $n=4$, donde la importancia aumenta en el decrecimiento de la escala, $m_{i}$ : Total de libros publicados del grupo $i$ en el período evaluado y $\operatorname{Libros}_{i j}$ : Participación del trabajador (profesor o investigador) en la publicación $j$ del grupo $i$ y se determina con la expresión siguiente:

$$
\text { Libros }_{i j}=\frac{1}{\text { cantidad de autores del Libro j del grupo i }}\left(f_{\text {conpiejicad externa }} / f_{\text {ayuda intema }}\right)
$$

- $O_{\text {ind }}$ : Producción en otros indicadores asociados a la actividad científica que la institución considere

$$
O_{\text {ind }}=\sum_{i=1}^{w} O t_{-} \text {ind }_{i} * P_{-} O t_{i}
$$

Donde $O t_{-}$ind $_{i}$ : Medición del indicador $i$ añadido en el investigador que puede ser importante para la organización como puede ser índice h, h10, RG, entre otros y $P_{-}$Otind $_{i}$ : Peso otorgado por la organización al indicador $i$.

Una vez establecida la métrica con sus relaciones cuantitativas por cada producción científica es importante explicar el procedimiento recomendado para la determinación de los pesos implicados en cada una de las expresiones presentadas en la sección anterior. Para ello es necesario precisar que los pesos dan una medida de la importancia relativa de cada elemento con respecto al resto (Medina \& García, 2016). Para el caso de los pesos que aparecen en la ecuación (1), los especialistas deben decidir la importancia de cada una de las producciones relacionadas con la actividad científica. Existen en la bibliografía diferentes formas de asignación de pesos, que pueden considerarse objetivos o subjetivos (Márquez Rosales, 1999; Penades Pla, 2017). En el caso de los objetivos se parte de las evaluaciones hechas a las alternativas en la matriz de evaluación para posteriormente, mediante métodos objetivos, deducir los pesos de cada criterio, dentro de este grupo se encuentran el método de la entropía y medidas de dispersión. Por su parte los subjetivos asignan primero los pesos y luego se deducen las evaluaciones. Dentro de este grupo se encuentran el método de asignación directa, el método ranking, el método Trade-offs y de comparación por pares. En este último se encuentra el método del autovector que es el utilizado en el Proceso de Análisis Jerárquico el cual se propone para utilizar en este artículo.

La razón principal por la cual se trabaja con el PAJ es debido a la forma de ponderación de criterios subjetivos y la opción de poder trasladar la realidad percibida a la escala de la razón, (Martínez, Gómez, Ibarra, \& Moncada, 2018; Smith, Gallego, \& Martínez, 2018), haciendo uso de la escala de valoración propuesta por Saaty (1987) en las comparaciones pareadas. Según Su Jeong, García Moruno, González Gómez, and Carver (2016) el Proceso Analítico Jerárquico (PAJ) es un método ampliamente aceptado, que constituye una aproximación eficaz para ex- 
traer los pesos de importancia relativa de los criterios en un problema dado. Para realizar las comparaciones entre cada par de atributo a ponderar se construye una matriz cuadrada, donde por filas y columnas aparecen estos atributos (A), el elemento aij de la matriz representa la comparación entre dos atributos utilizando la escala que aparece en la tabla 1. En esta matriz se cumple que. Los pesos de cada atributo Wj se calculan determinando el valor propio principal de la matriz ya sea de forma general o por cualquier método numérico (Márquez Rosales, 1999). Para ello se considera necesario utilizar el siguiente procedimiento:

Paso 1: Normalizar cada columna de la matriz de comparaciones pareadas dividiendo los elementos de cada columna por su suma.

Paso 2: Promediar por fila la matriz obtenida en el paso 1. Los valores obtenidos son una aproximación de los pesos buscados.

Por otra parte, el PAJ permite medir la inconsistencia de los juicios emitidos mediante la Proporción de Consistencia, que se expresa como el cociente entre el índice de Consistencia y el Índice Aleatorio, el mismo debe ser menor del $10 \%$.

Tabla \#1. Escala de Saaty

\begin{tabular}{|c|c|}
\hline Intensidad & Definición \\
\hline 1 & Importancia igual \\
\hline 3 & Importancia moderada \\
\hline 5 & Importancia fuerte \\
\hline 7 & Importancia muy fuerte \\
\hline 9 & Importancia extrema \\
\hline $2,4,6,8$ & Valores intermedios \\
\hline
\end{tabular}

Fuente: Penades Pla (2017)

Para la determinación en cada uno de los casos que ocupa al artículo se utilizará la herramienta informática implementada para el PAJ, el AHP Online System. Por tanto, se comenzará obteniendo los pesos de las diferentes producciones para la productividad científica.

Imagen 1. Matriz de comparaciones pareadas entre cada uno de los indicadores de la Producción Científica.

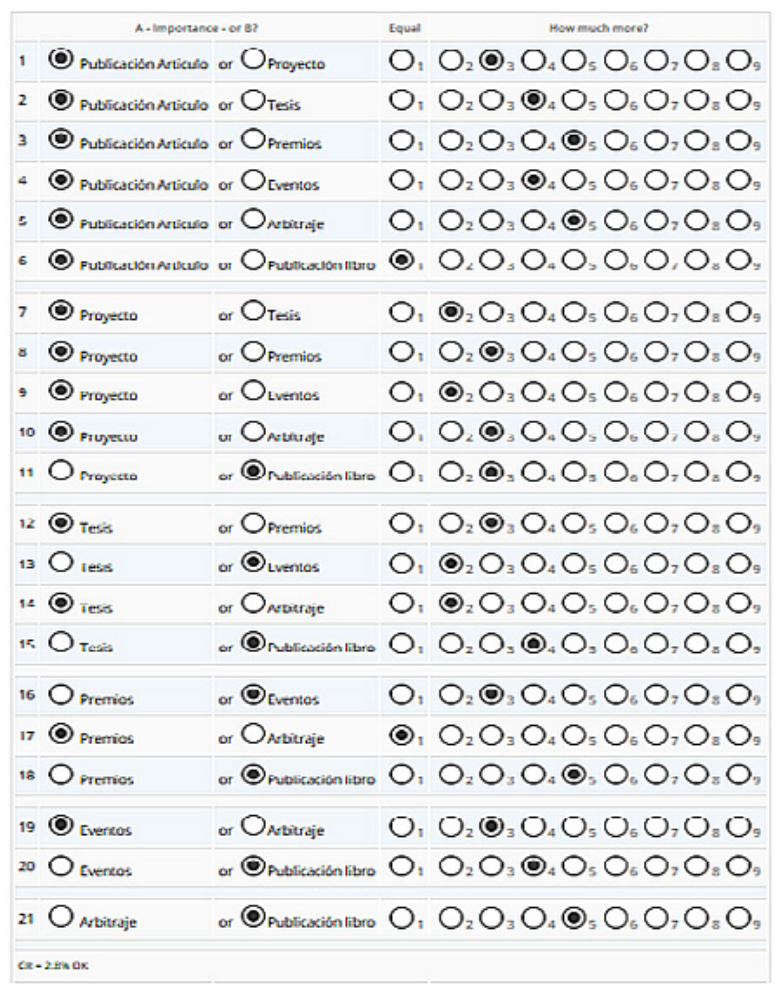

Fuente: Obtenida con AHP Online System. 
Imagen 2. Vector de preferencia y matriz de Saaty para los indicadores de la Producción Científica.

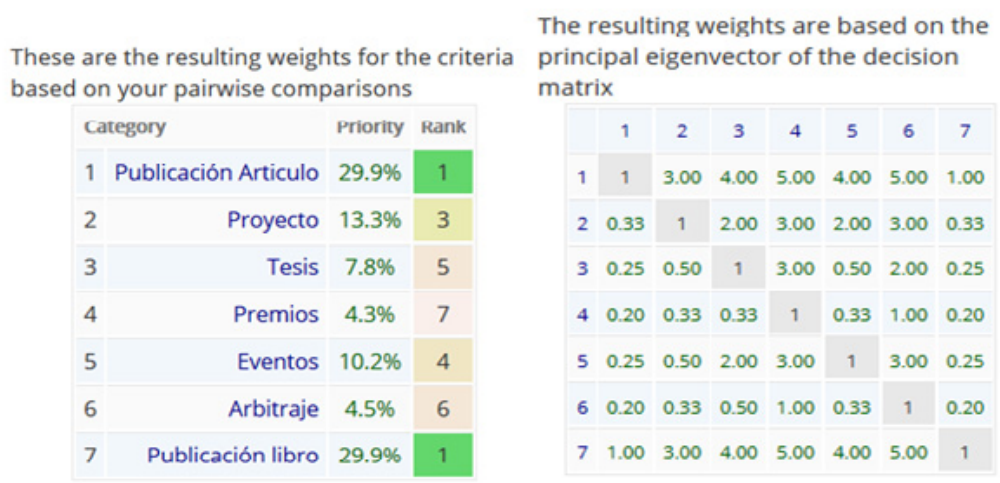

Fuente: Obtenida con AHP Online System.

El vector de peso $\left(P_{\text {public }}, P_{\text {proyec }}, P_{\text {tesis }}, P_{\text {premio }}, P_{\text {event }}, P_{\text {arb }}, P_{\text {lib }}, O_{\text {ind }}\right)$ será $(0.30,0.13,0.08,0.04,0.10,0.05,0.30)$

Para el caso de los tipos de publicaciones

Imagen 3. Matriz de comparaciones pareadas entre los diferentes Tipos de Publicaciones.

\begin{tabular}{|c|c|c|c|c|}
\hline \multicolumn{3}{|c|}{ A - Importance - or B? } & Equal & How much more? \\
\hline 1 & (C) grupo1 & or Ogrupo2 & $\mathrm{O}_{1}$ & $\mathrm{O}_{2} \mathrm{O}_{3} \mathrm{O}_{4} \mathrm{O}_{5} \mathrm{O}_{6} \mathrm{O}_{7} \mathrm{O}_{8} \mathrm{O}_{9}$ \\
\hline 2 & ( grupo1 & or $\bigcirc$ grupo3 & $\mathrm{O}_{1}$ & $\mathrm{O}_{2} \mathrm{O}_{3} \mathrm{O}_{4} \mathrm{O}_{5} \mathrm{O}_{6} \mathrm{O}_{7} \mathrm{O}_{8} \mathrm{O}_{9}$ \\
\hline 3 & - grupo1 & or Ogrupo4 & $\mathrm{O}_{1}$ & $\mathrm{O}_{2} \mathrm{O}_{3} \mathrm{O}_{4} \mathrm{O}_{5} \mathrm{O}_{6} \mathrm{O}_{7} \mathrm{O}_{8} \mathrm{O}_{9}$ \\
\hline 4 & - grupo2 & or O grupo3 & $\mathrm{O}_{1}$ & $\mathrm{O}_{2} \bigcirc_{3} \mathrm{O}_{4} \mathrm{O}_{5} \mathrm{O}_{6} \mathrm{O}_{7} \mathrm{O}_{8} \mathrm{O}_{9}$ \\
\hline 5 & (- grupo2 & or Ogrupo4 & $\mathrm{O}_{1}$ & $\mathrm{O}_{2} \mathrm{O}_{3} \mathrm{O}_{4} \mathrm{O}_{5} \mathrm{O}_{6} \mathrm{O}_{7} \mathrm{O}_{8} \mathrm{O}_{9}$ \\
\hline 6 & - grupo3 & or Ogrupo 4 & $\mathrm{O}_{1}$ & $\mathrm{O}_{2} \mathrm{O}_{3} \mathrm{O}_{4} \mathrm{O}_{5} \mathrm{O}_{6} \mathrm{O}_{7} \mathrm{O}_{8} \mathrm{O}_{9}$ \\
\hline
\end{tabular}

Fuente: Obtenida con AHP Online System.

Imagen 4. Vector de preferencia y matriz de Saaty para los diferentes Tipos de Publicaciones.

These are the resulting weights for the
based on your pairwise comparisons
\begin{tabular}{|l|l|l|l|l|}
\hline Category & Priority & Rank \\
\hline 1 & grupo1 & $53.8 \%$ & 1 \\
\hline 2 & grupo2 & $27.4 \%$ & 2 \\
\hline 3 & grupo3 & $12.8 \%$ & 3 \\
\hline 4 & grupo4 & $6.0 \%$ & 4 \\
\hline
\end{tabular}

The resulting weights are based
on the principal eigenvector of

the decision matrix

\begin{tabular}{|c|c|c|c|c|}
\hline & 1 & 2 & 3 & 4 \\
\hline 1 & 1 & 3.00 & 4.00 & 6.00 \\
\hline 2 & 0.33 & 1 & 3.00 & 5.00 \\
\hline 3 & 0.25 & 0.33 & 1 & 3.00 \\
\hline 4 & 0.17 & 0.20 & 0.33 & 1 \\
\hline
\end{tabular}

Fuente: Obtenida con AHP Online System.

El vector de peso será $(0.54,0.27,0.13,0.06)$

Para el caso de los proyectos 
Imagen 5. Matriz de comparaciones pareadas entre los diferentes Tipos de Proyectos.

\begin{tabular}{|c|c|c|c|c|}
\hline \multicolumn{3}{|c|}{ A-Importance - or B? } & Equal & How much more? \\
\hline 1 & (-) Proyectos internacionales & or OProyectos nacionales & $\mathrm{O}_{1}$ & $\mathrm{O}_{2} \mathrm{O}_{3} \bigcirc_{4} \mathrm{O}_{5} \mathrm{O}_{6} \mathrm{O}_{7} \mathrm{O}_{8} \mathrm{O}_{9}$ \\
\hline 2 & (-) Proyectos internacionales & or OProyectos empresariales & $\mathrm{O}_{1}$ & $\mathrm{O}_{2} \mathrm{O}_{3} \mathrm{O}_{4} \mathrm{O}_{5} \mathrm{O}_{6} \mathrm{O}_{7} \mathrm{O}_{8} \mathrm{O}_{9}$ \\
\hline 3 & - Proyectos internacionales & or Oproyectos institucionales & $\mathrm{O}_{1}$ & $\mathrm{O}_{2} \mathrm{O}_{3} \mathrm{O}_{4} \mathrm{O}_{5} \mathrm{O}_{6} \mathrm{O}_{7} \mathrm{O}_{8} \mathrm{O}_{9}$ \\
\hline 4 & (-) Proyectos nacionales & or Oproyectos empresariales & $\mathrm{O}_{1}$ & $\mathrm{O}_{2} \mathrm{O}_{3} \bigcirc_{4} \mathrm{O}_{5} \mathrm{O}_{6} \mathrm{O}_{7} \mathrm{O}_{8} \mathrm{O}_{9}$ \\
\hline 5 & (-) Proyectos nacionales & or OProyectos institucionales & $\mathrm{O}_{1}$ & $\mathrm{O}_{2} \mathrm{O}_{3} \mathrm{O}_{4} \mathrm{O}_{5} \mathrm{O}_{6} \mathrm{O}_{7} \mathrm{O}_{8} \mathrm{O}_{9}$ \\
\hline 6 & - Proyectos empresariales & or O Proyectos institucionales & $\mathrm{O}_{1}$ & $\mathrm{O}_{2} \bigcirc_{3} \mathrm{O}_{4} \mathrm{O}_{5} \mathrm{O}_{6} \mathrm{O}_{7} \mathrm{O}_{8} \mathrm{O}_{9}$ \\
\hline
\end{tabular}

Fuente: Obtenida con AHP Online System.

Imagen 6. Vector de preferencia y matriz de Saaty para los diferentes Tipos de Proyectos.

These are the resulting weights for the criteria

based on your pairwise comparisons

\begin{tabular}{|l|r|r|c|}
\hline Category & Priority & Rank \\
\hline 1 & Proyectos internacionales & $61.3 \%$ & 1 \\
\hline 2 & Proyectos nacionales & $22.7 \%$ & 2 \\
\hline 3 & Proyectos empresariales & $10.2 \%$ & 3 \\
\hline 4 & Proyectos institucionales & $5.8 \%$ & 4 \\
\hline
\end{tabular}

Fuente: Obtenida con AHP Online System.

El vector de peso será $(0.61,0.23,0.10,0.06)$

Para el caso de las tesis

Imagen 7. Matriz de comparaciones pareadas entre los diferentes Tipos de Tesis.

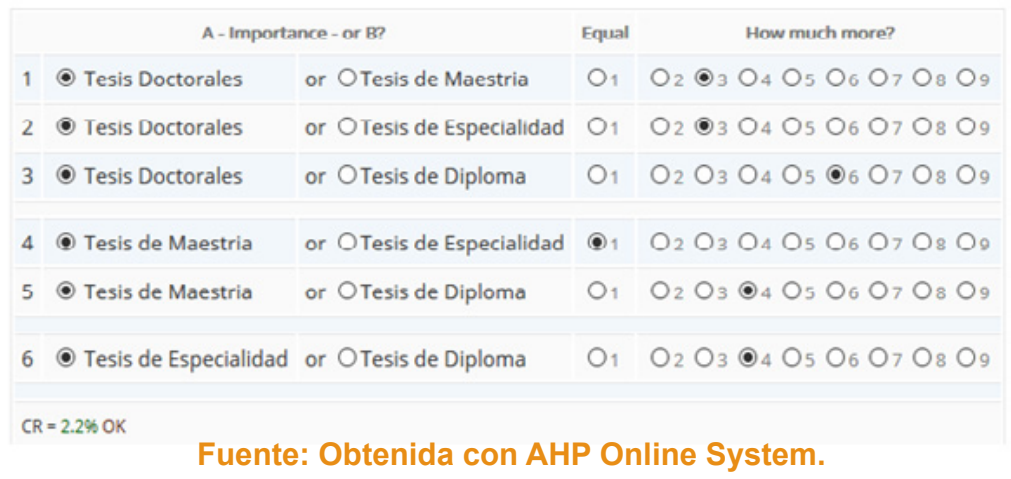


Imagen 8. Vector de preferencia y matriz de Saaty para los diferentes Tipos de Tesis.

These are the resulting weights for the criteri
bascd on your pairwisc comparisons
\begin{tabular}{|l|r|r|r|}
\hline Category & Priority & Rank \\
\hline 1 & Tesis Doctoralcs & $52.6 \%$ & 1 \\
\hline 2 & Tesis de Maestria & $20.6 \%$ & 2 \\
\hline 3 & Tesis de Especialidad & $20.6 \%$ & 2 \\
\hline 4 & Tesis de Diploma & $6.2 \%$ & 4 \\
\hline
\end{tabular}

The resulting weights are based on the principal eigenvector of the decision matrix

Fuente: Obtenida con AHP Online System.

El vector de peso será $(0.52,0.21,0.21,0.06)$

Para el caso de los premios

Imagen 9. Matriz de comparaciones pareadas entre los diferentes Tipos de Premios.

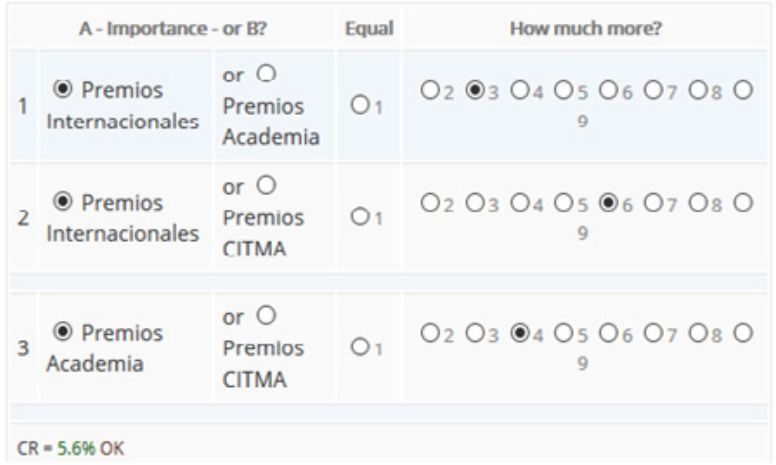

Fuente: Obtenida con AHP Online System.

Imagen 10. Vector de preferencia y matriz de Saaty para los diferentes Tipos de Premios.

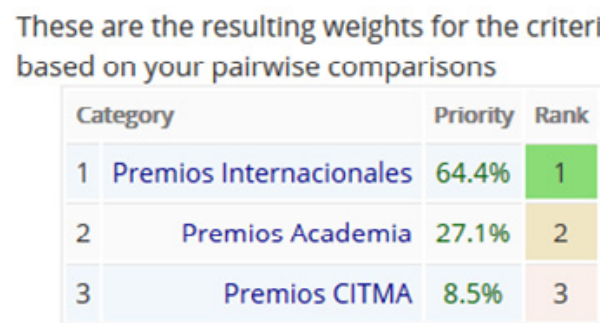

The resulting weights are based on the principal eigenvector of the decision matrix

\begin{tabular}{|c|c|c|c|}
\hline & 1 & 2 & 3 \\
\hline 1 & 1 & 3.00 & 6.00 \\
\hline 2 & 0.33 & 1 & 4.00 \\
\hline 3 & 0.17 & 0.25 & 1 \\
\hline
\end{tabular}

Fuente: Obtenida con AHP Online System.

Para el caso de los eventos 
Imagen 11. Matriz de comparaciones pareadas entre los diferentes Tipos de Eventos

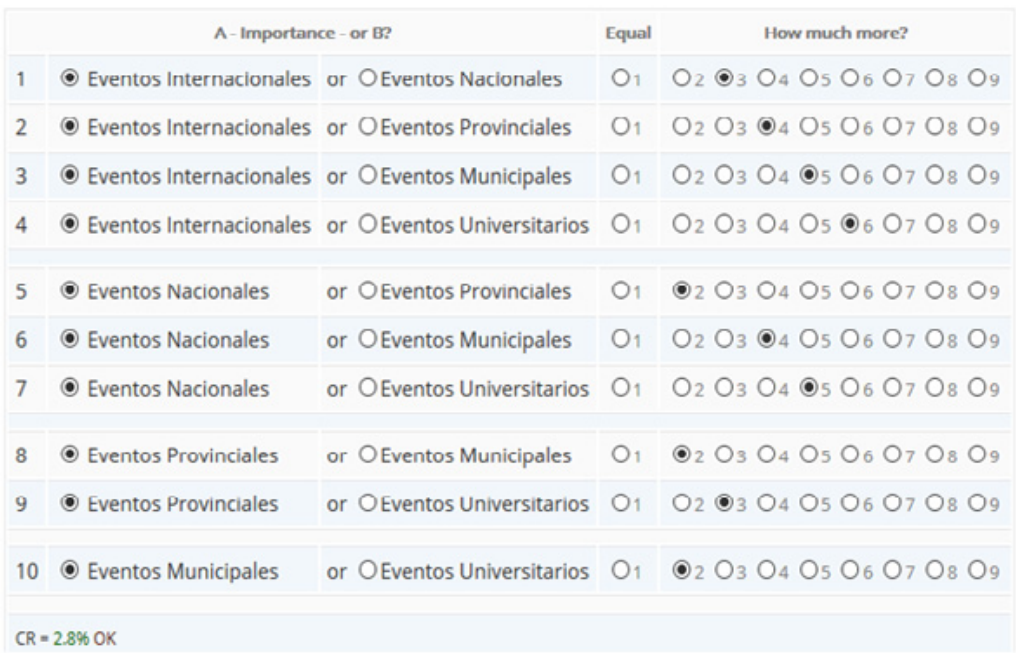

Fuente: Obtenida con AHP Online System.

Al obtener el Vector de preferencia y matriz de Saaty para los diferentes Tipos de Eventos de la misma manera que las anteriores se obtiene el vector de peso será $(0.48,0.25,0.14,0.08,0.05)$.

Una de las problemáticas para la medición de la ciencia es detectar la producción científica de los investigadores como bien se muestra en la bibliografía consultada (Aguillo Caño et al., 2017; Dorta-Contreras, 2018; Vessuri, 2013). En todos estos autores se toman los índices de visibilidad aportados por los perfiles de investigador; sin embargo, estos índices no siempre toman en cuenta todas las revistas en las cuales los autores publican ni todos los indicadores de la producción científica. La fiabilidad de los instrumentos de captación de la información juega un papel esencial, en el caso de Cuba, las universidades elaboran un documento oficial denominado Balance de Ciencia y Técnica que incluye todas las producciones científicas de la universidad, así como los premios obtenidos en diferentes niveles. De este documento puede extraerse la información necesaria para evaluar cuánto produce por cada autor pues se reflejan los artículos, premios, eventos, maestrías y doctorados. Otro documento a utilizar en el caso de Cuba es la evaluación de los profesores en la cual se colocan todas las actividades de carácter científico que han realizado que no aparecen en el Balance de Ciencia y Técnica como las tutorías de tesis de diploma, los tribunales de tesis de diploma, entre otras. Y, por último, el plan de trabajo del profesor, documento que expresa el tiempo dedicado por cada profesor a sus actividades en cada mes de trabajo. Este documento es importante pues permite delimitar cuánto tiempo dedica el profesor a las investigaciones científicas en su fondo de tiempo. De ello se puede inferir que el esfuerzo para obtener un resultado no debe ser evaluado de la misma manera en aquellos profesores que poseen poco tiempo dedicado a la investigación en comparación de aquellos que poseen mucho tiempo dedicado a la investigación.

\section{Metodología}

El método aplicado fue el análisis documental. Las universidades cubanas todos los años generan un documento denominado Balance de Ciencia y Técnica donde se plasman los resultados científicos de cada una de ellas: publicaciones, premios, patentes, tutorías de tesis, presentaciones de trabajos en eventos, entre otros. Cada uno de estos resultados posee los datos necesarios para aplicar la métrica y para ello se tomaron como objeto de revisión los Balances de Ciencia y Técnica del 2016, 2017 y 2018 de la Universidad de Matanzas. Posteriormente se realizó una aplicación parcial de la métrica tomando solamente la publicación de artículos. Del Balance de Ciencia y Técnica de los tres años se obtuvo un total de 598 investigadores que aparecen como autor de alguno de los artículos contenidos en ese documento. Se procedió a clasificar los artículos en los diferentes grupos de Ministerio de Educación Superior por cada año y a calcular la participación de cada autor en los artículos declarados. Con esa información se procedió a calcular la producción de artículos (PrA) en el período evaluado (2016-2018).

\section{Resultados}

Los resultados obtenidos serán mostrados en dos partes diferentes: una primera el análisis de los autores sin ponderar los artículos y otra con los artículos ponderados. Con artículos sin ponderar se obtiene que de los 597 profesores que declaran autores de artículos en el Balance de Ciencia y Técnica, solamente 10 profesores logran completar 3 artículos como total de sus participaciones en todos los grupos declarados por el MES. En la tabla se muestran otras comparaciones: 
Tabla \#2: Cantidad de profesores con más de tres artículos publicados

\begin{tabular}{|c|c|c|c|c|}
\hline Profesor & $\begin{array}{l}\text { Articulos Pu- } \\
\text { blicados }\end{array}$ & $\begin{array}{c}\text { Miembro del Consejo } \\
\text { Científico de la Universi- } \\
\text { dad }\end{array}$ & Doctor & $\begin{array}{l}\text { Miembro de Tribu- } \\
\text { nal Nacional }\end{array}$ \\
\hline$X X X X X X X X X$ & 3 & No pertenece & No & No \\
\hline$X X X X X X X X X$ & 6 & No pertenece & No & No \\
\hline$X X X X X X$ & 4 & No pertenece & Sí & Sí \\
\hline$X X X X X X X X$ & 3 & No pertenece & No & No \\
\hline$X X X X X X X$ & 24.8333333 & No pertenece & Sí & No \\
\hline$X X X X X X X X X$ & 3 & No pertenece & No & No \\
\hline$X X X X X X X X$ & 4.2 & No tiene datos & No se sabe & No \\
\hline$X X X X X X X X$ & 3 & Pertenece & Sí & No \\
\hline$X X X X X X X X X$ & 6.10952381 & Pertenece & Sí & Sí \\
\hline$X X X X X X X X X$ & 3 & No pertenece & No se sabe & No \\
\hline
\end{tabular}

Fuente: Elaboración de los autores.

De los datos obtenidos en la tabla se llegan a las siguientes inferencias:

- $70 \%$ no pertenece al Consejo Científico de la Universidad. Este indicador tiene dos lecturas, una primera que los miembros del Consejo Científico pudieran no poseer tiempo para publicar debido a una alta carga de reuniones. Una segunda lectura es que los actores principales de las publicaciones no fueron seleccionados para dirigir la ciencia en la universidad.

- $50 \%$ no es doctor: Este resultado demuestra que, como tendencia, un alto porcentaje de los profesores dejan de tener publicaciones después de haber concluido su formación doctoral

- 70\% no es miembro de Tribunal Nacional de Doctorado: Ídem al apartado de pertenencia al Consejo Científico.

Con artículos ponderados según el vector propuesto se obtiene que de los 597 profesores que declaran autores de artículos en el Balance de Ciencia y Técnica, solamente 19 profesores sobrepasan la unidad en todos los grupos declarados por el MES ponderados. En la tabla se muestran otras comparaciones:

Tabla \#3: Profesores con más de un artículo ponderado.

\begin{tabular}{|c|c|c|c|c|}
\hline $\begin{array}{c}\text { Publicados total } 3 \\
\text { articulos ponde- } \\
\text { rado }\end{array}$ & Total General & $\begin{array}{c}\text { Miembro del CC } \\
\text { Universidad }\end{array}$ & Doctor & $\begin{array}{l}\text { Miembro de } \\
\text { Tribunal Nacional }\end{array}$ \\
\hline $\mathrm{XXXXXXXX}$ & 1.08 & No & Sí & No \\
\hline XXXXXXXX & 1.215 & No & Sí & No \\
\hline$\overline{X X X X X X X X}$ & 1.62 & No & No & No \\
\hline XXXXXXXX & 3.24 & No & No & No \\
\hline XXXXXXXX & 2.16 & Sí & Sí & Sí \\
\hline XXXXXXXX & 1.62 & No & No & No \\
\hline XXXXXXXX & 1.35 & No & Sí & Sí \\
\hline XXXXXXXX & 15.03 & No & Sí & No \\
\hline XXXXXXXX & 1.08 & No & Sí & No \\
\hline XXXXXXXX & 1.62 & No & No & No \\
\hline XXXXXXXX & 2.268 & No & No & No \\
\hline XXXXXXXX & 1.62 & Sí & Sí & No \\
\hline XXXXXXXX & 1.08 & No & No & No \\
\hline XXXXXXXX & 1.125 & No & No & No \\
\hline XXXXXXXX & 3.29914286 & Sí & Sí & Sí \\
\hline
\end{tabular}




\begin{tabular}{|l|c|c|c|c|}
\hline$X X X X X X X X$ & 1.38214286 & Sí & Sí & Sí \\
\hline$X X X X X X X X$ & 1.116 & Sí & Sí & Sí \\
\hline$X X X X X X X X$ & 1.62 & No & No & No \\
\hline$X X X X X X X X$ & 1.08 & Sí & Sí & . \\
\hline
\end{tabular}

Fuente: Elaboración de los autores.

De los datos obtenidos en la tabla se llegan a las siguientes inferencias:

- $48 \%$ son doctores: Ello indica que, aunque se mantiene la tendencia en la cantidad de artículos, en este caso los doctores aportan calidad a las publicaciones que poseen.

- 69 \% no pertenece al Consejo Científico: Se mantiene la tendencia ya expuesta antes per en este caso es preocupante pues estamos hablando de la calidad de los artículos que se producen. Otra lectura es a la inversa, el Consejo Científico de la Universidad posee solamente un $10 \%$ de sus miembros que sobrepasa en 1 el indicador de publicaciones con calidad, cuestión esta que puede ser preocupante en términos de publicaciones.

- 74 \% no pertenece a Tribunales Nacionales: Idem al anterior, sobre todo porque la Universidad de Matanzas posee un total de 19 Miembros de Tribunales Nacionales.

Del análisis del documento Balance de Ciencia y Técnica se obtienen las siguientes insuficiencias:

- Necesidad de uniformidad de las formas de citación en los balances que permita distinguir a los investigadores: las diversas formas de citación omiten determinadas partes del nombre de los autores, cuestión esta que no permite apreciar quiénes son los autores. Esta situación se agrava cuando existen autores con nombres y apellidos parecidos que tiene a confundir.

- Declaración por áreas del conocimiento de los principales resultados del balance para atender a la especificidad de cada una: cada área del conocimiento posee complejidades inherentes a su forma de proceder para obtener los resultados científicos, ello complejiza la aplicación de la métrica para todos los profesores.

- Necesidad de revisar las clasificaciones de las revistas pues existen artículos reportados de grupo I que realmente no lo son y de clasificar los resultados por áreas del conocimiento: Cada área del conocimiento posee especificidades propias que impactan en las condiciones y formas de investigar. La clasificación por áreas del conocimiento permite precisar con mayor claridad la validez del trabajo de los investigadores al poderse comparar con colegas de su mismo perfil.

De los resultados obtenidos expuestos hasta el momento se puede inferir:

- Disminución de la productividad científica en la universidad, aunque se constata un aumento en su calidad.

- Poca representatividad de los actores principales de la ciencia en los consejos donde se trazan las políticas acerca de esta.

- Incremento de la productividad científica en profesores antes de la defensa de doctorados y maestrías que después de obtenida decrecen.

- Profesores sin línea de investigación definida que poseen artículos sobre temáticas diferentes en cada año. Pirámide de investigación con base estrecha, lo cual hace que uno o dos doctores atiendan un único aspirante.

\section{Conclusiones}

Los procesos de medición de la productividad científica son de interés de las organizaciones pues permiten reorganizar su capital humano en función de los procesos de investigación. Cada organización universitaria puede considerar diversos indicadores y, al mismo tiempo, asignarle valores a cada uno de ellos. La métrica propuesta cuantifica las relaciones contenidas en el proceso de evaluación de la productividad científica que incluye los principales indicadores usados por las universidades cubanas. La métrica se comporta mayormente como una función aditiva de multiplicación de los pesos por la sumatoria de las participaciones inversamente proporcional al tiempo que la persona dedica a la investigación.

\section{Bibliografía}

Aguillo Caño, I. F., Uribe Tirado, A., \& López López, W. (2017). Visibilidad de los investigadores colombianos según sus indicadores en Google Scholar y ResearchGate. Diferencias y similitudes con la clasificación oficial del sistema nacional de ciencia-COLCIENCIAS. Revista Interamericana de Bibliotecología, 40(3), 221-230. doi:10.17533/ udea.rib.v40n3a03.

Aguillo, I. F. (2015). Metrics 2.0 for a Science 2.0. Paper presented at the Science 2.0, Hamburg.

Biava, L., Pagani, C., \& de Oliveira, G. C. (2019). Indicadores de pesquisa científica como ferramenta para a gestão da universidade. Brazilian Applied Science Review, 3(1), 69-91. 
Caballero-Uribe, C. V., \& Viloria-Doria, J. C. (2018). Un análisis del impacto del nuevo modelo de clasificación de revistas científicas según Colciencias. Salud Uninorte, 34(1), vii-xii.

Conacyt. (s/f). Índice de Revistas Mexicanas de Divulgación Científica y Tecnológica. Retrieved from https://www. conacyt.gob.mx/index.php/comunicacion/indice-de-revistas-mexicanas-de-divulgacion-cientifica-y-tecnologica

Deusto, B. U. d. (2018). Criterios específicos de valoración según campo de conocimiento 2018: 7. CCSS, Políticas, Comportamiento, Educación. Retrieved from http://biblioguias.biblioteca. deusto.es/c.php?g=667892\&p=4737358

Dorta-Contreras, A. J. (2018). Ciencia a la medida. Estudios bibliométricos y cienciométricos en una nueva sección. Revista Habanera de Ciencias Médicas, 17(4), 508-509.

Ferretti, F., Pereira, Â. G., Vértesy, D., \& Hardeman, S. (2018). Research excellence indicators: time to reimagine the 'making of'? Science and Public Policy, 45(5), 731-741. doi:10.1093/scipol/scy007.

Gorraiz, J. (2018). Los mil y un reflejos de las publicaciones en el laberinto de espejos de las nuevas métricas. El profesional de la información, 27(2), 231-236.

Gumpenberger, C., Glänzel, W., \& Gorraiz, J. (2016). The ecstasy and the agony of the altmetric score. Scientometrics, 108(2), 977-982.

Handley Jones, M. R. (2017). Can research quality be measured quantitatively? On quality of scholarship, numerical research indicators and academic publishing - experiences from Norway. Fennia - International Journal of Geography, 195(2), 164-174. doi:10.11143/fennia.66602

Kosten, J. (2016). A classification of the use of research indicators. Scientometrics, 108(1), 457-464. doi:10.1007/ s11192-016-1904-7

Manzano-Arrondo, V. (2017). Hacia un cambio paradigmático para la evaluación de la actividad científica en la Educación Superior. Revista de la Educación Superior, 46(183), 1-35. doi:10.1016/j.resu.2017.08.003

Márquez Rosales, H. (1999). Métodos matemáticos de evaluación de factores de riesgo para el Patrimonio Arqueológico: una aplicación GIS del método de jerarquías analíticas de TL Saaty. Spal, 6, 21-37.

Martínez, R. E., Gómez, J. C. O., Ibarra, D. E., \& Moncada, C. A. L. (2018). Selección de una infraestructura de medición inteligente de energía usando una técnica de decisión multicriterio. Scientia et Technica, 23(2), 136-142.

Medina, B. M., \& García, M. R. (2016). Aplicación de métodos de decisión multicriterio discretos al análisis de alternativas en estudios informativos de infraestructuras de transporte. Pensamiento Matemático, 6(2), 27-45.

Ortega, J. L. (2018). Reliability and accuracy of altmetric providers: a comparison among Altmetric.com, PlumX and Crossref Event Data. Scientometrics, 116(3), 2123-2138.

Penades Pla, V. (2017). Aplicación de la toma de decisión multicriterio al diseño sostenible de puentes de hormigón.

Smith, D., Gallego, C. A. G., \& Martínez, M. (2018). Análisis multi-criterio para evaluar la capacidad de absorción de energía de tubos fabricados con láminas de metal expandido y sólidas. Revista UIS Ingenierías, 17(1), 69-80.

Su Jeong, J., García Moruno, L., González Gómez, D., \& Carver, S. (2016). Implementación de un método para alcanzar un paisaje resiliente mediante la ubicación apropiada de edificaciones rurales. Economía Agraria y Recursos Naturales, 16(1380-2016-115492), 19.

Vessuri, H. (2013). De la transferencia a la creatividad. Los papeles culturales de la ciencia en los países subdesarrollados. Polis. Revista Latinoamericana, 1-23. 


\section{Dados dos autores}

Walfredo González Hernández

Doctor en Ciencias Pedagógicas y Profesor Titular de la Carrera Ingeniería Informática. Graduado de Licenciatura en Educación Especialidad Matemática - Computación en 1995, obtiene su maestría en Didáctica de la Matemática en 2003 y su doctorado en 2004. Ha publicado más de 40 artículos en revistas arbitradas e indexadas, dirige proyectos internacionales y es árbitro de varias revistas y eventos.

walfredo.glez@umcc.com

\section{Maritza Petersson Roldán}

Recibió de Licenciatura en Matemáticas, en 1985, la maestría en Técnicas de Optimización y apoyo a la decisión en 1999 y el doctorado en 2006. Es profesora titular en el Departamento de Matemáticas. Investiga en Técnicas de modelación y estadísticas de procesos y servicios productivos y la valoración económica.

maritza.petersson@umcc.cu

\section{Yonelkys Ramos Mena}

Graduado de Ingeniería Informática de la Universidad de Matanzas em el año 2017. Actualmente es professor de la carrera Ingeniería Informática en la Universidad de Matanzas. Doctorante del área de comercio electrónico. yonelkys.ramos@umcc.cu

Received: 2019-03-15

Accepted: 2020-03-29

\section{(c) $)$ EY}

This work is licensed under a Creative Commons Attribution 4.0 United States License.

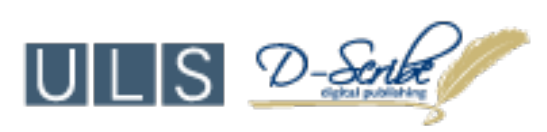

This journal is published by the University Library System of the University of Pittsburgh as part of its D-Scribe Digital Publishing Program and is cosponsored by the University of Pittsburgh Press 